\title{
ON A TYPE OF SASAKIAN SPACE
}

\author{
M. C. CHAKI and D. GHOSH
}

(Received 12 November 1969)

Communicated by B. Mond

\section{Introduction}

A Sasakian space [1] $M^{n}(n=2 m+1)$ is a Riemannian $n$-space with a positive definite metric tensor $g_{i j}$ and a unit Killing vector field $\eta$ which satisfies

$$
\eta_{j, k l}=\eta_{k} g_{l j}-\eta_{j} g_{l k}
$$

where the comma denotes covariant differentiation with respect to the metric tensor. In a recent paper [2] M. C. Chaki and A. N. Roy Chowdhury studied conformally recurrent spaces of second order, or briefly conformally 2-recurrent spaces, that is, non-flat Riemannian spaces $V_{n}(n>3)$ defined by

$$
C_{k j i, l m}^{h}=a_{l m} C_{k j i}{ }^{h}
$$

where $C_{k j i}{ }^{h}$ is the conformal curvature tensor:

$$
\begin{aligned}
C_{k j i}{ }^{h}= & R_{k j i}{ }^{h}-\frac{1}{n-2}\left(g_{j i} R_{k}{ }^{h}-g_{k i} R_{j}^{h}+R_{j i} \delta_{k}{ }^{h}-R_{k i} \delta_{j}{ }^{h}\right) \\
& +\frac{R}{(n-1)(n-2)}\left(g_{j i} \delta_{k}{ }^{h}-g_{k i} \delta_{j}{ }^{h}\right)
\end{aligned}
$$

and $a_{l m}$ is a tensor not identically zero.

The present paper deals with conformally 2-recurrent Sasakian spaces, that is, Sasakian spaces in which (2) is satisfied. It is proved that such an $n$-space $(n>3)$ is of constant curvature.

\section{Some formulas in a Sasakian Space}

Since in a Sasakian space $\eta$ is a unit vector field

$$
\eta^{r} \eta_{r}=1
$$

Applying Ricci's identity to $\eta_{j}$ we obtain

$$
\eta_{j, k l}-\eta_{j, l k}=-\eta_{r} R_{l k j}^{r}
$$

508 
where $R_{k j i}{ }^{h}$ is the Riemannian curvature tensor:

$$
R_{k j i}{ }^{h}=\frac{\partial}{\partial x^{k}}\left\{\begin{array}{l}
h \\
j i
\end{array}\right\}-\frac{\partial}{\partial x^{j}}\left\{\begin{array}{l}
h \\
k i
\end{array}\right\}+\left\{\begin{array}{c}
h \\
k r
\end{array}\right\}\left\{\begin{array}{l}
r \\
j i
\end{array}\right\}-\left\{\begin{array}{l}
h \\
j r
\end{array}\right\}\left\{\begin{array}{c}
r \\
k i
\end{array}\right\}
$$

Using (1) we can express (1.2) as

$$
\eta_{r} R_{l k j}^{r}=\eta_{l} g_{j k}-\eta_{k} g_{j l}
$$

Contracting (1.3) with $g^{j l}$ we have

$$
\eta_{r} R_{k}^{r}=(n-1) \eta_{k}
$$

Thus in a Sasakian space the formulas (1.1), (1.3) and (1.4) hold.

\section{Conformally 2-recurrent Sasakian space}

Let us suppose that a Sasakian space is conformally 2-recurrent. If possible, let a conformally 2-recurrent Sasakian space be not conformally flat. It has been proved in theorem 1 of [2] that if a conformally 2-recurrent space with positive definite metric is not conformally flat, then its tensor of recurrence $a_{l m}$ is symmetric. Hence if a conformally 2-recurrent Sasakian space be not conformally flat, then

$$
C_{k j i, l m}{ }^{h}-C_{k j i, m l}{ }^{h}=0
$$

Applying Ricci's identity to the left-hand side of $(2.1)$ we get

$$
C_{k j i}{ }^{r} R_{m l r}{ }^{h}-C_{k j r}{ }^{h} R_{m l i}{ }^{r}-C_{k r i}{ }^{h} R_{m l j}{ }^{r}-C_{r j i}{ }^{h} R_{m l k}{ }^{r}=0
$$

Transvecting this with $\eta_{h} \eta^{m}$ and using (1.1) and (1.3) we have

$$
\begin{aligned}
C_{k j i l}-\eta_{r} \eta_{l} C_{k j i}{ }^{r}+\eta_{r} \eta_{i} C_{k j l}{ }^{r}+\eta_{j} \eta_{r} C_{k l i}^{r} & \\
& +\eta_{r} \eta_{k} C_{l j i}^{r}+g_{l j} \eta^{r} \eta_{s} C_{r k i}^{s}-g_{l k} \eta^{r} \eta_{s} C_{r j i}{ }^{s}=0
\end{aligned}
$$

Contracting this with $g^{i j}$ we get

$$
\eta^{r} \eta_{s} C_{r k i}^{s}=0
$$

Substituting this value in $(2.2)$ we have

$$
C_{k j i l}-\eta_{r} \eta_{l} C_{k j i}^{r}+\eta_{r} \eta_{i} C_{k j l}^{r}+\eta_{j} \eta_{r} C_{k l i}^{r}+\eta_{k} \eta_{r} C_{l j i}^{r}=0
$$

Now,

$$
\left.C_{k j i}^{r} \eta_{r}=\frac{1}{n-2}\left\{\left(\frac{R}{n-1}-1\right) \eta_{k} g_{j i}-\eta_{j} g_{k i}\right)-\left(\eta_{k} R_{j i}-\eta_{j} R_{k i}\right)\right\}
$$

Using (2.5) we can express (2.4) as 


$$
C_{k j i l}+\frac{1}{n-2}\left[\left(\frac{R}{n-1}-1\right)\left(\eta_{k} g_{j l}-\eta_{j} g_{k l}\right)-\left(\eta_{k} R_{j l}-\eta_{j} R_{k l}\right)\right] \eta_{i}=0
$$

Contracting this with $g^{i k}$ we get

$$
R_{l j}=\left(\frac{R}{n-1}-1\right) g_{l j}+\left(n-\frac{R}{n-1}\right) \eta_{l} \eta_{j}
$$

Substituting this value in (2.6) we have $C_{k j i l}=0$. But this is contrary to hypothesis.

Hence if a Sasakian space is conformally 2-recurrent, then it is conformally flat. It has been proved by Okumura [3] that a conformally flat Sasakian space is of constant curvature. We can therefore state the following theorem.

THEOREM. A conformally 2-recurrent Sasakian space is of constant curvature.

In theorem 9 of [2], it has been proved that every $n$-dimensional $(n>3)$ projective 2-recurrent space, that is a Riemannian space in which Weyl's projective curvature tensor:

$$
W_{k j i}{ }^{h}=R_{k j i}{ }^{h}-\frac{1}{n-1}\left(\delta_{k}{ }^{h} R_{j i}-\delta_{j}{ }^{h} R_{k i}\right)
$$

satisfies the relation $W_{k j i}{ }^{h},{ }_{l m}=a^{\prime}{ }_{l m} W_{k j i}{ }^{h}$ for a non-zero tensor $a_{l m}^{\prime}$, is a conformally 2 -recurrent space. We have therefore the following corollary of the above theorem:

COROLLARY. A projective 2-recurrent Sasakian space is of constant curvature.

\section{References}

[1] S. Sasaki, Lecture note on almost contact manifolds Tohoku University (1965).

[2] M. C. Chaki and A. N. Roy Chowdhury, 'On conformally recurrent Spaces of Second order', Journ. Australian Math. Soc. 10 (1969), 155-161.

[3] M. Okumura, 'Some remarks on space with a certain contact structure', Tohoku Math. J. 14 (1962), $135-145$.

Department of Pure Mathematics

Calcutta University 